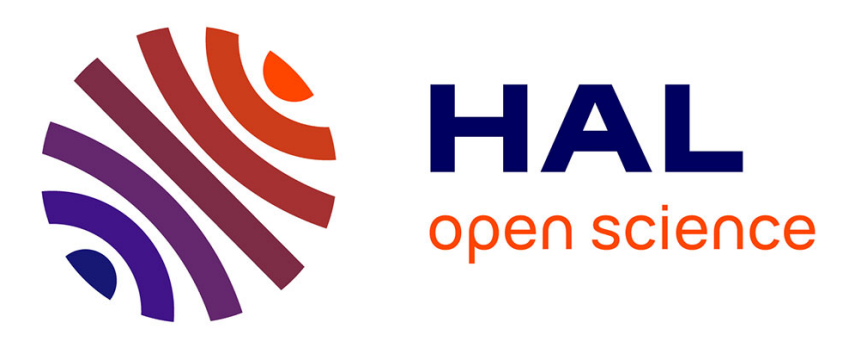

\title{
Multimodal Groups' Analysis for Automated Cohesion Estimation
}

\author{
Lucien Maman
}

\section{To cite this version:}

Lucien Maman. Multimodal Groups' Analysis for Automated Cohesion Estimation. ICMI '20: INTERNATIONAL CONFERENCE ON MULTIMODAL INTERACTION, Oct 2020, Virtual Event, Netherlands. pp.713-717, 10.1145/3382507.3421153 . hal-03187452

\section{HAL Id: hal-03187452 https://hal.science/hal-03187452}

Submitted on 26 Nov 2021

HAL is a multi-disciplinary open access archive for the deposit and dissemination of scientific research documents, whether they are published or not. The documents may come from teaching and research institutions in France or abroad, or from public or private research centers.
L'archive ouverte pluridisciplinaire HAL, est destinée au dépôt et à la diffusion de documents scientifiques de niveau recherche, publiés ou non, émanant des établissements d'enseignement et de recherche français ou étrangers, des laboratoires publics ou privés. 


\title{
Multimodal Groups' Analysis for Automated Cohesion Estimation
}

\author{
Lucien Maman \\ lucien.maman@telecom-paris.fr \\ LTCI, Télécom Paris, Institut polytechnique de Paris \\ Palaiseau, 91120, France
}

\begin{abstract}
Groups are getting more and more scholars' attention. With the rise of Social Signal Processing (SSP), many studies based on Social Sciences and Psychology findings focused on detecting and classifying groups' dynamics. Cohesion plays an important role in these groups' dynamics and is one of the most studied emergent states, involving both group motions and goals. This $\mathrm{PhD}$ project aims to provide a computational model addressing the multidimensionality of cohesion and capturing its subtle dynamics. It will offer new opportunities to develop applications to enhance interactions among humans as well as among humans and machines.
\end{abstract}

\section{CCS CONCEPTS}

- Computing methodologies $\rightarrow$ Machine learning; • Humancentered computing;

\section{KEYWORDS}

Cohesion; Computational Model; Dataset; Emergent State; Multimodality

\section{ACM Reference Format:}

Lucien Maman. 2020. Multimodal Groups' Analysis for Automated Cohesion Estimation. In Proceedings of the 2020 International Conference on Multimodal Interaction (ICMI '20), October 25-29, 2020, Virtual event, Netherlands. ACM, New York, NY, USA, 5 pages. https://doi.org/10.1145/3382507.3421153

\section{INTRODUCTION}

Groups are getting more and more scholars' attention. With the rise of Social Signal Processing (SSP), many studies based on Social Sciences and Psychology findings focused on detecting and classifying groups' dynamics [21].

Emergent states play an important role in these group dynamics. These are social processes that result from the micro-level affective, behavioral and cognitive interactions among group members, through the micro-processes of group interaction (e.g., [19, 32]). Cohesion is one of the most studied emergent states [38], involving both group emotions [30] and goals [23]. Nevertheless, several definitions and theoretical models of cohesion exist, limiting the comparisons across studies [9, 39]. Over the last decade, scholars focused on how to automatically detect and enhance cohesion but were either relating to a simplistic definition of cohesion or not

ICMI '20, October 25-29, 2020, Virtual event, Netherlands

(C) 2020 Association for Computing Machinery.

This is the author's version of the work. It is posted here for your personal use. Not for redistribution. The definitive Version of Record was published in Proceedings of the 2020 International Conference on Multimodal Interaction (ICMI '20), October 25-29, 2020, Virtual event, Netherlands, https://doi.org/10.1145/3382507.3421153. addressing its subtle nuances and dynamics. Moreover, they suffered from a lack of publicly available data specifically designed for cohesion. The end-goal of this $\mathrm{PhD}$ project is to provide a computational model addressing the multidimensionality of cohesion and capturing its dynamics. It will offer new opportunities to develop applications to enhance interactions among humans as well as among humans and machines (e.g. virtual agents and robots).

\section{BACKGROUND AND RELATED WORK}

\subsection{Theoretical models}

In the 1940s, Lewin introduced the first definition of cohesion, inspired by the field theory [24]. He referred to it as" $a$ group characteristic that depends on its size, organization and intimacy" [25]. Over time, scholars suggested diverse definitions and models to describe this emergent state $[2,12,28,43]$ and the 2-dimensional model introduced by Carron became widely used [8]. More recently, Severt and Estrada [40] proposed an integrative framework, taking into account Carron's model and other researchers' ideas and improvements (i.e., $[3,4,10,15])$. This framework is structured into two functional properties: the affective one and the instrumental one. The former refers to all the aspects that highlight the emotional impact on a group member and, by extension, the group as a whole (e.g., behaviors or elements of an interaction such as cooperation or exchange). The latter one corresponds to "those aspects that highlight the goal-and task-based activities of the group" [40]. Each functional property is separated into two facets (interpersonal and group pride, and social and task, respectively) for which we can distinguish two levels: horizontal and vertical.

\subsection{Automated approaches to detect cohesion}

Nonverbal communication has been shown to be a more powerful predictor of group-level cohesion than verbal behavior [20]. Among the computational studies interested in predicting cohesion, the ones focusing on small groups' nonverbal cues usually yield better results than the ones focusing on verbal communication. Similarly, studies using a multimodal approach generally obtained better performances compare to the ones using uni-modal models. In a pioneering study, Hung and Gatica-Perez integrated both audio and video non-verbal features, exploring cohesion through multiple dimensions in meetings context [17]. They also collected annotations of cohesion provided by external observers to establish a reference for evaluating their model. Nanninga and colleagues recently extended this work, integrating pairwise and group features related to the alignment of para-linguistic speech behavior [34]. They proved that their audio-based features were more effective at predicting the social dimension of cohesion, with respect to the baseline set in [17]. Both studies defined cohesion prediction as 
a binary classification problem (i.e., positive or negative). They, however, did not focus on how the task and social dimensions are related to each other. Furthermore, they did not take other phenomena such as leadership or social cognition into account to improve cohesion prediction. With the evidence that cohesion is related to other phenomena $[1,6,22,26]$, integrating features linked to other phenomena in computational models could lead to a better understanding of cohesion and its dynamics. Other studies investigated cohesion at a longitudinal level (e.g., for a period of 20 working days or within a 4-months simulation of a space exploration mission context), with the use of sociometric badges (e.g., [35, 47]). All the features collected through these sociometric badges were, however, only based on individuals and no group level features were collected.

\subsection{Cohesion in a virtual environment}

Virtual Reality (VR) applications can be used to teach and improve social skills (e.g., $[18,36]$ ), suggesting that interactions in a virtual environment share similarities with the real-world environment. At present, however, only a few studies compared interactions in both VR and real-world settings (e.g., [41, 42]). In [46], Widerström et al. designed an experiment consisting of a collaborative puzzle-solving task, carried out by two persons in virtual and real environments to study leadership and performance in the two settings. They found that, in both settings, participants agreed on the leadership and the degree of collaboration, suggesting that the perception of this emergent state is similar in a real and a virtual environment. This study, however, did not focus on the dynamics of these phenomena and only explored dyadic interactions. To the best of my knowledge, there is no research designed for studying cohesion, at a group level, that includes both virtual and real-world settings. Such study would help to gain insight on how cohesion emerges and varies in a virtual environment with respect to a real-world environment.

\section{RESEARCH QUESTIONS}

This $\mathrm{PhD}$ project is aimed at building a computational model able to predict the dynamics of cohesion and to capture the relationships between its social and task dimensions. My research will specifically focus on the social and task dimensions at the horizontal level, based on Severt and Estrada's framework [40] as it aligns with the dominant approach in the current teams' literature (e.g., $[1,6,22])$. Furthermore, no automated study of cohesion was ever based on this framework, even though it provides a finer level of categorization than other models (e.g., Carron's model [8]). It also acknowledges and integrates that cohesion is a dynamic phenomenon that can be expressed in various ways depending on the context. To achieve the goal of this $\mathrm{PhD}$ project, the focus will be laid on the following research questions:

RQ1: Which multimodal socio-behavioral features are relevant to predict cohesion and the relationships between social and task dimensions? How these features can be coded to feed a computational model?

Intuitively, we could expect that both social and task dimensions are expressed differently, through various modalities, as they serve distinct purposes (quantifying social bonds and quantifying task commitment, respectively). Psychological models (e.g., [8, 40]), however, assume that theses dimensions are not orthogonal, meaning that there may be behavioral correlates which are indicative for both dimensions. Previous approaches do not take into account these relationships. The context in which the group is interacting also plays an important role in the group cohesion. Past research, however, mainly focused on a meeting scenario where, for example, full-body movements are restricted. Moreover, no innate group features have been developed. Features are either based on dyadic or individual features that are then aggregated to form a group feature. As the goal is to detect the dynamics of cohesion at a group level, it is important to develop a set of group and individual multimodal features shared by both dimensions.

RQ2: How the knowledge on other cognitive and behavioral phenomena can be explicitly modeled to structure a computational model of cohesion?

To the best of my knowledge, no study interested in automatically predicting cohesion has integrated any other cognitive or behavioral phenomena (e.g., motivation, leadership, performance, emotions, social cognition) in their model. As stated in Severt and Estrada's study, various links and relationships between cohesion and other phenomena may be observed depending on the function (e.g., instrumental), the facet (e.g., social) or the level of analysis of cohesion (e.g., horizontal) that is being investigated. With this in mind, the focus will be on integrating the leadership and social cognition phenomena into the computational model's architecture. The cohesion-leadership link has already been proved (e.g., [27]) and would give more insights on the interaction while warmth and competence (i.e., social cognition) could help to give some extra contextual information regarding the group's members perceptions.

RQ3: What computational architectures can be envisaged to predict cohesion and its dynamics?

Most of the computational studies related to cohesion rely on different definitions, making it difficult to compare findings across studies. Moreover, these studies developed Machine Learning models to predict the presence or the absence of cohesion for the social and task dimensions, separately, without investigating the relationships between cohesion's dimensions over time as well as with other group phenomena. It highlights the need to develop a comprehensive computational model to address these comments.

RQ4: How does virtual cohesion could improve collaboration in multimodal systems?

New paradigms of group interaction could emerge with the advent of new technologies and the actual world context (e.g., health crisis, climate change) as more and more tools are developed to encourage people to meet and gather virtually (e.g. ICMI2020 as a virtual conference). Understanding how cohesion manifests in both virtual and real environments would provide another angle of research and enrich our comprehension of cohesion, leading to the development of more robust multimodal systems. 


\section{RESEARCH PLAN AND METHODOLOGY}

This research is conducted in four stages within three years, with the following proposed scope, methodology, and time frames.

\section{Stage 1: Investigating the foundations of cohesion}

Understanding the concept of cohesion and being aware of the different methods used to assess it (e.g., questionnaires or coding schemes) is a key part of the project as scientific knowledge from Social Science and Psychology will drive and inform research on the computational model. The first months of the project were dedicated to (1) conduct a comprehensive literature review on cohesion covering both Psychology and Computer Science approaches, (2) choose the most suitable multidimensional theoretical model of cohesion that acknowledge relationships between its dimensions and with other phenomena and (3) choose a well-established questionnaire that can assess and measure various dimensions of cohesion. As a result of these investigations, my $\mathrm{PhD}$ project will focus on the instrumental property of cohesion at a horizontal level based on Severt and Estrada's model of cohesion [40]. This model integrates the most accepted ideas from the Psychological literature on cohesion and acknowledges the multidimensionality of cohesion. It also suggests that some relationships exist between its dimensions and with other phenomena, highlighting the need for exploring their impact on cohesion. The GEQ questionnaire [8] will be used to measure self-assessments of group cohesion (i.e., its social and task dimensions). This questionnaire is widely used and several studies have shown how the GEQ can be leveraged for addressing group situations in different contexts (e.g., [7, 11, 16, 33]).

\section{Stage 2: Collecting a multimodal dataset for cohesion and group analysis}

The second stage, which consisted of designing and performing a data collection to capture the dynamics of cohesion, ran throughout the end of the first year. It also included multiple rounds of pre-tests, the recruitment of the participants and the post-processing of the data. The data collection involved six other researchers from four different laboratories (see [31] for more details). We focused our efforts on capturing the variations of cohesion (i.e., increase or decrease), in the context of an escape game (i.e., a social game). Social games have been considered as a viable research methodology to address the subtle nuances of human-human communication by several research domains (e.g., [5, 14, 37, 44]). This stage aimed to collect the first multimodal dataset (containing audio, video and MoCap data) specifically designed for the study of cohesion dynamics. Besides, we also gathered self-assessments of cohesion, participants' emotional state and perceived leadership and warmth and competence through the use of well-established questionnaires.

\section{Stage 3: Building a computational model}

This stage aims at designing the model in such a way that it will: (1) follow theoretical sociological models, (2) integrate the multidimensional nature of cohesion, (3) take the temporality and the relationships between its dimensions over time into account and (4) consider the impact of other phenomena on cohesion. In order to address these goals, the first step consists of defining a set of multimodal nonverbal features that will feed the computational model. The challenge is to define a common set of features that will be used to detect changes in the dynamics as well as some dimension-specific features that would help to refine the prediction for each dimension in order to reflect the fact that social and task dimensions are not orthogonal. Afterwards, how to capture the dynamics of cohesion should be addressed. As a first approximation, the focus will be to address such dynamics as an increase or decrease of cohesion. This problem can be tackled as a binary classification task (e.g., Increase/Decrease) or a multiclass classification task (e.g., Increase/Stable/Decrease). A more refined step will consist of addressing dynamics as a regression task (e.g., by how much the cohesion varied). At this point, we could imagine running several Machine Learning models in parallel, or sequentially, to independently predict each dimension. Another option would be to design a multiclass model to predict both dimensions at the same time. Finally, the features related to the other phenomena could be used at multiple layers: as part of the feature layer (input) and/or as part of a "context" layer that would come on top of the feature layer to augment the input features by using additional information extracted from these phenomena.

\section{Stage 4: Evaluating the computational model}

The evaluation of the computational model will be performed by comparing the output of the model with the annotations provided by the participants and by external raters. These two kinds of annotations will be used in order to yield a "true" assessment of cohesion by minimizing the known biases introduced by self-assessment (e.g., drifting off the ratings towards socially desirable characteristics) and external assessment (e.g., problems in the attribution of characteristics) [45]. The two last stages are expected to be iterative over the last two years of the $\mathrm{PhD}$ program. The features will first be computed and then incrementally adjusted. We will have a similar approach for the overall architecture of the computational model and its implementation as the plan is to integrate various novelties.

\section{RESULTS AND CONTRIBUTIONS TO DATE}

\subsection{Collection of the GAME-ON dataset}

GAME-ON is composed of five tasks designed to elicit variations (i.e., increase or decrease) of the social and task dimensions of cohesion, following Severt and Estrada's framework of cohesion [40]. The GEQ questionnaire [8] was administered after each task in order to observe variations of cohesion all along with the data collection. More than 11 hours of multimodal data have been recorded. After post-processing, GAME-ON [31] now contains data of 15 groups of three participants without any interruption or missing data. Figure 1 shows three participants posing and their corresponding 17-points skeleton obtained with the MoCap system.

Statistical analysis, including Friedman tests and post-hoc Conover's tests with a Bonferroni correction, has been conducted based on the cohesion scores. These scores are computed per participant and per dimension (i.e., social and task) from the six self-assessments collected. The analysis confirmed that we successfully managed to control the variations of cohesion in the expected directions (increase or decrease) for the social dimension and four transitions over five regarding the task dimension. 


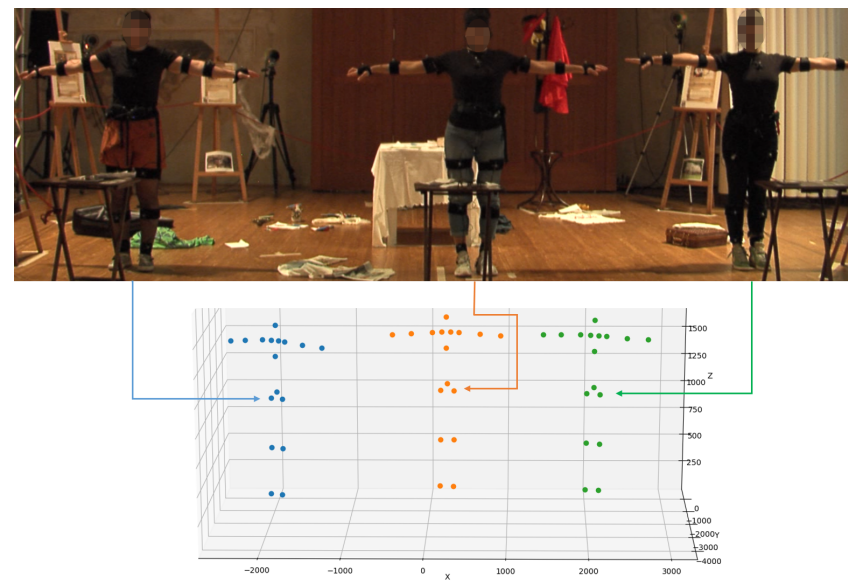

Figure 1: Example taken from the GAME-ON dataset showing a group posing during the data collection and the three corresponding 17-points skeletons.

\subsection{Ongoing studies}

The two first stages are now completed and a first exploration of the data led to the following ongoing studies:

Setting up a baseline for predicting decreases of cohesion using MoCap-based features and self-assessments of cohesion and assessing features impact and importance on the model. First, a set of 14 group features and their respective mean, standard deviation, max, min and skewness, were computed. They are either calculated at an individual level and then aggregated or computed at a group level (see Table 1). Then, we produced the labels (increase

Table 1: Description of the aggregated and innate nonverbal group features used to predict decreases of cohesion.

\begin{tabular}{|c|c|}
\hline Aggregated group features & Innate group features \\
\hline Max distance between group members & Facing balance \\
Spacial association of max distance & Participation equality \\
Amount of hand gestures while not walking & Turn-taking freedom \\
Average amount of walking & Number of floor exchanges \\
Average posture expansion & Presence of F-Formation \\
Posture difference in group & \\
Time touch is detected & \\
Time someone is being faced & \\
Movement difference in group & \\
\hline
\end{tabular}

or decrease) by taking the mean rank difference between two consecutive GEQ scores. With a multilabel setting and using a Random Forest classifier, we reached $64 \% \pm 3 \%$ and $67 \% \pm 3 \%$ classification accuracy and $64 \% \pm 3 \%$ and $67 \% \pm 3 \%$ F1-scores for task and social dimensions, respectively. We employed a repeated nested 10 -fold cross-validation with five repetitions across all slices to validate our model. Cross-folds were randomly generated and stratified over the set of groups and tasks. The number of pruned decision trees and their maximum depth were estimated using grid search on a 5-fold cross-validation. The best model was selected using the highest average accuracy over both social and task dimension and used pruned decision trees with a maximum depth of 5 , a total of 100 estimators, and Gini imbalance evaluation criterion. Finally, we provided a method based on notions from cooperative game theory (i.e., SHAP values [29]) to assess the feature set impact and importance on our setting.

Exploring the cohesion-social cognition relationships. In addition to participants' self-assessment of cohesion, GAMEON also comprises both self and external assessments of the two major dimensions of social cognition: Warmth and Competence (W\&C) [13]. The first phase consisted of exploring what were the correlations between the GEQ and the W\&C questionnaires. The next step is now to develop multimodal features related to $\mathrm{W} \& \mathrm{C}$, based on the previous analysis and psychological findings (e.g., kinetic energy and amplitude of arm movements for W\&C, respectively). Then, we should ensure that these features are correlated to the W\&C questionnaire. Finally, observing their correlations with the GEQ questionnaire would strengthen the link between these phenomena.

\section{FUTURE WORK AND CONTRIBUTIONS}

As part of the iterative process presented in stage 3 of Section 4, the baseline for detecting changes in the dynamics of cohesion will continue to be improved by integrating other multimodal features (with a focus on audio and video modalities). A particular effort will be made in order to design the computational model's architecture so that it will integrate the relationships between the social and task dimensions as well as with the other phenomena. As part of the last stage of Section 4, an external annotation campaign will be run. The goal is to see if there is an agreement between self and external ratings in order to study how to combine them to limit or avoid the biases introduced in each type of label. Finally, a pilot study will be designed to compare cohesion in both real and virtual environments. In addition to the existing contributions previously mentioned, the following major contributions are envisioned:

(1) Advancing our understanding of cohesion by providing a set of features able to capture the dynamics of cohesion that are shared between social and task dimensions of cohesion.

(2) Providing a computational model able to capture the relationship between social and task dimensions of cohesion and to predict the dynamics of cohesion.

(3) Running a pilot study to compare cohesion in both real and virtual environments.

\section{ACKNOWLEDGMENTS}

I would like to thank my supervisors (Prof. Giovanna Varni, Prof. Mohamed Chetouani and Prof. Laurence Likforman-Sulem) for all their feedback and guidance and Fabian Walocha for working with me and under my supervision on developing the features and the models for the first ongoing study, as part of his Master's thesis internship. This work has been partially supported by the French National Agency (ANR) in the frame of its Technological Research JCJC program (GRACE, project ANR-18-CE33-0003-01, funded under the Artificial Intelligence Plan). 


\section{REFERENCES}

[1] Bryan P Acton, Michael T Braun, and Roseanne J Foti. 2019. Built for unity: assessing the impact of team composition on team cohesion trajectories. Fournal of Business and Psychology (2019), 1-16.

[2] Kurt W Back. 1951. Influence through social communication. The fournal of Abnormal and Social Psychology 46, 1 (1951), 9-23.

[3] Daniel J Beal, Robin R Cohen, Michael J Burke, and Christy L McLendon. 2003 Cohesion and Performance in Groups: A Meta-Analytic Clarification of Construct Relations. Fournal of Applied Psychology 88, 6 (2003), 989-1004.

[4] Kenneth A Bollen and Rick H Hoyle. 1990. Perceived cohesion: A conceptual and empirical examination. Social forces 69, 2 (1990), 479-504.

[5] Clara Bonillo, Teresa Romão, and Eva Cerezo. 2019. Persuasive games in Interactive Spaces: The Hidden Treasure Game. In Proceedings of the XX International Conference on Human Computer Interaction. 1-8.

[6] Michael T Braun, Steve WJ Kozlowski, Tara A Brown, and Richard P DeShon 2020. Exploring the Dynamic Team Cohesion-Performance and Coordination-Performance Relationships of Newly Formed Teams. Small Group Research (2020).

[7] Sally A Carless and Caroline De Paola. 2000. The measurement of cohesion in work teams. Small group research 31, 1 (2000), 71-88.

[8] Albert V Carron, W Neil Widmeyer, and Lawrence R Brawley. 1985. The development of an instrument to assess cohesion in sport teams: The Group Environment Questionnaire. Journal of Sport and Exercise psychology 7, 3 (1985), 244-266.

[9] Chris W Coultas, Tripp Driskell, C Shawn Burke, and Eduardo Salas. 2014. A conceptual review of emergent state measurement: Current problems, future solutions. Small Group Research 45, 6 (2014), 671-703.

[10] Kenneth L Dion. 2000. Group Cohesion: From Field of Forces to Multidimensional Construct. Group Dynamics: Theory, Research, and Practice 4, 1 (2000), 7-26.

[11] Paul A Estabrooks and Albert V Carron. 2000. The Physical Activity Group Environment Questionnaire: An instrument for the assessment of cohesion in exercise classes. Group Dynamics 4, 3 (2000), 230-243.

[12] Leon Festinger, Stanley Schachter, and Kurt Back. 1950. Social pressures in informal groups; a study of human factors in housing. (1950).

[13] Susan T Fiske, Amy JC Cuddy, and Peter Glick. 2007. Universal Dimensions of Social Cognition: Warmth and Competence. Trends in cognitive sciences 11, 2 (2007), 77-83.

[14] Gili Freedman and Mary Flanagan. 2017. From dictators to avatars: Furthering social and personality psychology through game methods. Social and personality psychology compass 11, 12 (2017), e12368.

[15] James Griffith. 1988. Measurement of group cohesion in US Army units. Basic and applied social psychology 9, 2 (1988), 149-171.

[16] Jean-Philippe Heuzé and Paul Fontayne. 2002. Questionnaire sur l'Ambiance du Groupe: A French-Language Instrument for Measuring Group Cohesion. Journal of Sport and Exercise Psychology 24, 1 (2002), 42-67.

[17] Hayley Hung and Daniel Gatica-Perez. 2010. Estimating Cohesion in Small Groups Using Audio-Visual Nonverbal Behavior. IEEE Transactions on Multimedia 12, 6 (2010), 563-575.

[18] Fengfeng Ke and Tami Im. 2013. Virtual-reality-based social interaction training for children with high-functioning autism. The fournal of Educational Research 106, 6 (2013), 441-461.

[19] Steve WJ Kozlowski. 2015. Advancing research on team process dynamics: Theoretical, methodological, and measurement considerations. Organizational Psychology Review 5, 4 (2015), 270-299.

[20] Uliyana Kubasova, Gabriel Murray, and McKenzie Braley. 2019. Analyzing Verbal and Nonverbal Features for Predicting Group Performance. In Proc. Interspeech 2019. ISCA, 1896-1900.

[21] Nale Lehmann-Willenbrock, Hayley Hung, and Joann Keyton. 2017. New frontiers in analyzing dynamic group interactions: Bridging social and computer science. Small group research 48, 5 (2017), 519-531.

[22] Francisco M Leo, Inmaculada González-Ponce, Tomás García-Calvo, David Sánchez-Oliva, et al. 2019. The relationship among cohesion, transactive memory systems, and collective efficacy in professional soccer teams: A multilevel structural equation analysis. Group Dynamics: Theory, Research, and Practice 23, 1 (2019), 44-56.

[23] Dani Levine, Daphna Buchsbaum, Kathy Hirsh-Pasek, and Roberta M Golinkoff. 2019. Finding events in a continuous world: A developmental account. Developmental psychobiology 61, 3 (2019), 376-389.

[24] Kurt Lewin. 1939. Field theory and experiment in social psychology: Concepts and methods. American journal of sociology 44, 6 (1939), 868-896.

[25] Kurt Lewin. 1946. Behavior and development as a function of the total situation. In Manual of child psychology. John Wiley \& Sons Inc, 791-844.

[26] David Lyle Light Shields, Douglas E Gardner, Brenda Jo Light Bredemeier, and Alan Bostro. 1997. The Relationship Between Leadership Behaviors and Group Cohesion in Team Sports. The fournal of Psychology 131, 2 (1997), 196-210.

[27] David Lyle Light Shields, Douglas E Gardner, Brenda Jo Light Bredemeier, and Alan Bostro. 1997. The relationship between leadership behaviors and group cohesion in team sports. the fournal of Psychology 131, 2 (1997), 196-210.
[28] Albert J Lott and Bernice E Lott. 1965. Group cohesiveness as interpersonal attraction: A review of relationships with antecedent and consequent variables. Psychological bulletin 64, 4 (1965), 259-309.

[29] Scott M Lundberg and Su-In Lee. 2017. A unified approach to interpreting model predictions. In Advances in neural information processing systems. 4765-4774.

[30] Joe C Magee and Larissa Z Tiedens. 2006. Emotional ties that bind: The roles of valence and consistency of group emotion in inferences of cohesiveness and common fate. Personality and Social Psychology Bulletin 32, 12 (2006), 1703-1715.

[31] Lucien Maman, Eleonora Ceccaldi, Nale Lehmann-Willenbrock, Laurence Likforman-Sulem, Mohamed Chetouani, Gualtiero Volpe, and Giovanna Varni. 2020. GAME-ON: A Multimodal Dataset for Cohesion and Group Analysis. IEEE Access 8 (2020), 124185-124203.

[32] Michelle A Marks, John E Mathieu, and Stephen J Zaccaro. 2001. A temporally based framework and taxonomy of team processes. Academy of management review 26, 3 (2001), 356-376.

[33] Michael D Michalisin, Steven J Karau, and Charnchai Tangpong. 2004. Top management team cohesion and superior industry returns: An empirical study of the resource-based view. Group \& Organization Management 29, 1 (2004), $125-140$.

[34] Marjolein C Nanninga, Yanxia Zhang, Nale Lehmann-Willenbrock, Zoltán Szlávik, and Hayley Hung. 2017. Estimating Verbal Expressions of Task and Social Cohesion in Meetings by Quantifying Paralinguistic Mimicry. In Proceedings of the 19th ACM International Conference on Multimodal Interaction. Association for Computing Machinery, 206--215.

[35] Daniel Olguín-Olguín and Alex Pentland. 2010. Sensor-based organisational design and engineering. International fournal of Organisational Design and Engineering 1, 1-2 (2010), 69-97.

[36] Kyung-Min Park, Jeonghun Ku, Soo-Hee Choi, Hee-Jeong Jang, Ji-Yeon Park, Sun I Kim, and Jae-Jin Kim. 2011. A virtual reality application in role-plays of social skills training for schizophrenia: a randomized, controlled trial. Psychiatry research 189, 2 (2011), 166-172.

[37] Elizabeth Redcay and Leonhard Schilbach. 2019. Using second-person neuroscience to elucidate the mechanisms of social interaction. Nature Reviews Neuroscience 20, 8 (2019), 495-505.

[38] Lisa Rosh, Lynn R Offermann, and Rhonda Van Diest. 2012. Too close for comfort? Distinguishing between team intimacy and team cohesion. Human Resource Management Review 22, 2 (2012), 116-127.

[39] Eduardo Salas, Rebecca Grossman, Ashley M Hughes, and Chris W Coultas. 2015. Measuring team cohesion: Observations from the science. Human factors 57, 3 (2015), 365-374.

[40] Jamie B Severt and Armando X Estrada. 2015. On the function and structure of group cohesion. In Team cohesion: Advances in psychological theory, methods and practice. Vol. 17. Emerald Group publishing limited, 3-24.

[41] Mel Slater, Amela Sadagic, Martin Usoh, and Ralph Schroeder. 2000. Small-group behavior in a virtual and real environment: A comparative study. Presence: Teleoperators \& Virtual Environments 9, 1 (2000), 37-51.

[42] Juliana Sutanto, Chee Wei Phang, Chuan Hoo Tan, and Xianghua Lu. 2011. Dr. Jekyll vis-a-vis Mr. Hyde: Personality variation between virtual and real worlds. Information \& management 48, 1 (2011), 19-26.

[43] Annie Van Bergen and J Koekebakker. 1959. "Group cohesiveness" in laboratory experiments. Acta Psychologica 16 (1959), 81-98.

[44] Eric Van Dijk, Carsten KW De Dreu, and Jörg Gross. 2020. Power in economic games. Current opinion in psychology 33 (2020), 100-104

[45] Alessandro Vinciarelli and Gelareh Mohammadi. 2014. A survey of personality computing. IEEE Transactions on Affective Computing 5, 3 (2014), 273-291.

[46] Josef Wideström, Ann-Sofie Axelsson, Ralph Schroeder, Alexander Nilsson, Ilona Heldal, and Åsa Abelin. 2000. The collaborative cube puzzle: a comparison of virtual and real environments. In Proceedings of the third international conference on Collaborative virtual environments. 165-171.

[47] Yanxia Zhang, Jeffrey Olenick, Chu-Hsiang Chang, Steve WJ Kozlowski, and Hayley Hung. 2018. TeamSense: assessing personal affect and group cohesion in small teams through dyadic interaction and behavior analysis with wearable sensors. Proceedings of the ACM on Interactive, Mobile, Wearable and Ubiquitous Technologies 2, 3 (2018), 1-22. 